\title{
Adaptive Algorithm for Estimation of Two-Dimensional Autoregressive Fields from Noisy Observations
}

\begin{abstract}
Alimorad Mahmoudi
Electrical Engineering Department, Shahid Chamran University, Ahvaz, Iran

Correspondence should be addressed to Alimorad Mahmoudi; a.mahmoudi@scu.ac.ir

Received 26 September 2014; Revised 4 December 2014; Accepted 11 December 2014; Published 25 December 2014

Academic Editor: Nikolai N. Leonenko

Copyright ( 2014 Alimorad Mahmoudi. This is an open access article distributed under the Creative Commons Attribution License, which permits unrestricted use, distribution, and reproduction in any medium, provided the original work is properly cited.

This paper deals with the problem of two-dimensional autoregressive (AR) estimation from noisy observations. The Yule-Walker equations are solved using adaptive steepest descent (SD) algorithm. Performance comparisons are made with other existing methods to demonstrate merits of the proposed method.
\end{abstract}

\section{Introduction}

The problem of two-dimensional (2D) autoregressive modeling is very important in many signal processing applications. This problem has applications in image processing, radar, sonar, and communications. In image processing, it has been applied to image modeling [1], texture analysis [2], and hyperspectral imagery [3]. In radar and sonar, it can be included in direction finding, model based detection, and spectral estimation [4-6]. It also can be applied to fading channel estimation in communications [7].

In noise-free case, 2D AR estimation is investigated in [810]. A $2 \mathrm{D}$ lattice structure is proposed for $2 \mathrm{D}$ AR modeling in [8] which is capable of simultaneously providing all possible types of $2 \mathrm{D}$ causal quarter-plane (QP) and asymmetric halfplane (ASHP) AR models. Model identification of a noncausal 2D AR process is presented in [9]. An order recursive algorithm is proposed to solve 2D Yule-Walker equations. Modeling of 2D AR processes with various regions of support is considered in [10].

The one-dimensional AR parameter estimation from noisy observations is well investigated in the literatures (see [11-14]). The literature is insufficient for two-dimensional noisy autoregressive fields. In [15], a method based on combination of the Yule-Walker equations and third order moment is proposed. In this method, the driving noise process is forced to be non-Gaussian. Recently, in [16], combinations of the low order and high order Yule-Walker equations are solved as a quadratic eigenvalue problem. This method is an extension of the method proposed by Davila in [11].

In this paper, we propose a Yule-Walker based method for the estimation of 2D AR parameters from noisy observations. Due to the observation noise variance, the Least-Squares (LS) estimate of the parameters is biased. We propose an estimation scheme using the additional Yule-Walker equation beyond the order of the model for the observation noise variance estimation. Then, by steepest descent (SD) algorithm, we compensate bias from the estimation. Numerical examples are given to show the effectiveness of the proposed estimation method.

\section{Problem Formulation}

A 2D autoregressive (AR) field is given by

$$
x(m, n)=\sum_{\substack{i=0 \\(i, j) \neq(0,0)}}^{p_{1}} \sum^{p_{2}} a(i, j) x(m-i, n-j)+u(m, n),
$$

where $a(0,0)=1,\left(p_{1}, p_{2}\right)$ is the order of field, and $x(m, n)$ is output of the model, and the driving input field, $u(m, n)$, is a $2 \mathrm{D}$ white zero-mean stationary field having variance of $\sigma_{u}^{2}$. In practice, the observed field is given by

$$
y(m, n)=x(m, n)+v(m, n),
$$


where $v(m, n)$ is the observation noise of zero-mean stationary field having variance of $\sigma_{v}^{2}$ and it is assumed to be uncorrelated with $u(m, n)$.

The model is assumed causal and stable and with quarterplane (QP) support. The order of model $\left(p_{1}, p_{2}\right)$ is assumed to be known. Some order selection methods in $2 \mathrm{D}$ AR case are presented in [17].

The power spectral density (PSD) corresponding to noiseless 2D AR field in (1) is given by [18]

$$
S\left(w_{1}, w_{2}\right)=\frac{\sigma_{u}^{2}}{\left|1-\sum_{k=0}^{p_{1}} \sum_{\substack{l=0 \\(k, l) \neq(0,0)}}^{p_{2}} a(k, l) e^{-\left(i w_{1} k+i w_{2} l\right)}\right|^{2}} .
$$

The Yule-Walker (YW) equations for noiseless 2D AR field given by (1) are as follows [19]:

$$
\begin{array}{r}
\sum_{i=0}^{p_{1}} \sum_{j=0}^{p_{2}} a(i, j) r_{x}(k-i, l-j)=r_{x}(k, l)+\sigma_{u}^{2} \delta(k, l) \\
k \geq 0, \quad l \geq 0, \quad(i, j) \neq(0,0),
\end{array}
$$

where $r_{x}(k, l)=E\{x(m, n) x(m-k, n-l)\}$ is $2 \mathrm{D}$ autocorrelation function, $E$ is the expectation operator, and $\delta(k, l)$ is Kronecker delta function, defined by

$$
\delta(k, l)= \begin{cases}1 & (k, l)=(0,0) \\ 0 & (k, l) \neq(0,0)\end{cases}
$$

Due to uncorrelatedness of $u(m, n)$ and $v(m, n)$, we have $E\{u(m, n) v(m, n)\}=0 \forall m, n$. So, the autocorrelation function of the observed field is given by

$$
r_{y}(k, l)=r_{x}(k, l)+\sigma_{v}^{2} \delta(k, l) .
$$

Our objective is to estimate $a(i, j)$ for $i=0, \ldots, p_{1}, j=$ $0, \ldots, p_{2},(i, j) \neq(0,0)$ from the observations $y(m, n)$ for $(m=1, \ldots, M, n=1, \ldots, N)$.

\section{The Algorithm}

The YW equations in (4) can be written in a matrix form as

$$
\overline{\mathbf{R}}_{y} \overline{\mathbf{a}}=\sigma_{v}^{2} \overline{\mathbf{a}}+\sigma_{u}^{2}\left(\begin{array}{c}
\mathbf{b}_{\mathbf{0}} \\
\mathbf{0} \\
\vdots \\
\mathbf{0}
\end{array}\right),
$$

where $\overline{\mathbf{a}}=\left(\begin{array}{c}\mathbf{a}_{0} \\ \mathbf{a}_{1} \\ \vdots \\ \mathbf{a}_{p_{1}}\end{array}\right)$,

$$
\begin{aligned}
\overline{\mathbf{R}}_{y} & =\left[\begin{array}{cccc}
\mathbf{R}_{y}(0) & \mathbf{R}_{y}(-1) & \cdots & \mathbf{R}_{y}\left(-p_{1}\right) \\
\mathbf{R}_{y}(1) & \mathbf{R}_{y}(0) & \cdots & \mathbf{R}_{y}(-p+1) \\
\vdots & \vdots & \ddots & \vdots \\
\mathbf{R}_{y}\left(p_{1}\right) & \mathbf{R}_{y}\left(p_{1}-1\right) & \cdots & \mathbf{R}_{y}(0)
\end{array}\right], \\
\mathbf{R}_{y}(i) & =\left[\begin{array}{cccc}
r_{y}(i, 0) & r_{y}(i,-1) & \cdots & r_{y}\left(i,-p_{2}\right) \\
r_{y}(i, 1) & r_{y}(i, 0) & \cdots & r_{y}\left(i,-p_{2}+1\right) \\
\vdots & \vdots & \ddots & \vdots \\
r_{y}\left(i, p_{2}\right) & r_{y}\left(i, p_{2}-1\right) & \cdots & r_{y}(i, 0)
\end{array}\right],
\end{aligned}
$$

$\mathbf{a}_{i}=\left[\begin{array}{c}a(i, 0) \\ a(i, 1) \\ \vdots \\ a\left(i, p_{2}\right)\end{array}\right]$, and $\mathbf{b}_{\mathbf{0}}=\left[\begin{array}{c}1 \\ 0 \\ \vdots \\ 0\end{array}\right]$ are the $\left(p_{2}+1\right) \times 1$ vectors.

Because $\sigma_{u}^{2}$ is unknown, the first row in (7) is removed and after rearranging the equations in terms of a, the YW equations can be rewritten as follows:

$$
\mathbf{R}_{y} \mathbf{a}-\sigma_{v}^{2} \mathbf{a}=\mathbf{r}_{y}
$$

where $\mathbf{R}_{y}$ is $\left(p_{1} p_{2}+p_{1}+p_{2}\right) \times\left(p_{1} p_{2}+p_{1}+p_{2}\right)$ matrix, $\mathbf{r}_{y}$, and $\mathbf{a}$ are $\left(p_{1} p_{2}+p_{1}+p_{2}\right) \times 1$ vectors. Note that $\mathbf{a}$ is $\overline{\mathbf{a}}$ after removing the first element $a(0,0)$.

Multiplying both sides of (9) by $\mathbf{R}_{y}^{-1}$, we obtain

$$
\mathbf{a}=\mathbf{a}_{\mathrm{LS}}+\sigma_{v}^{2} \mathbf{R}_{y}^{-1} \mathbf{a} .
$$

If $\sigma_{v}^{2}$ is assumed to be known, we can iteratively estimate a using SD algorithm as follows [20]:

$$
\mathbf{a}^{(j)}=\mathbf{a}^{(j-1)}+\mu\left(\mathbf{a}_{\mathrm{LS}}+\sigma_{v}^{2} \mathbf{R}_{y}^{-1} \mathbf{a}^{(j-1)}-\mathbf{a}^{(j-1)}\right),
$$

where $\mathbf{a}_{\mathrm{LS}}=\mathbf{R}_{y}^{-1} \mathbf{r}_{y}$ is the Least-Squares (LS) estimate of $\mathbf{a}$, $\mu$ is the step size parameter, and $\mathbf{r}_{y}$ and $\mathbf{R}_{y}$ can be estimated from the observations. We can also control the stability and rate of convergence of the algorithm by changing $\mu$. The above equation converges if $\mu$ is selected between zero and $2 /(1-$ $\left.\lambda_{\min }\right)$ where $\lambda_{\min }$ is the minimum eigenvalue of the $\sigma_{v}^{2} \mathbf{R}_{y}^{-1}$ matrix.

In many signal processing applications, the observation noise variance is unknown, so we must estimate it. In the following subsection, we present a method to estimate the observation noise variance.

3.1. The Observation Noise Variance Estimation Given a. Consider the $\mathrm{YW}$ equations for lag $p_{1}+1$; we have

$$
\left[\begin{array}{llll}
\mathbf{R}_{y}\left(p_{1}+1\right) & \mathbf{R}_{y}\left(p_{1}\right) & \cdots & \mathbf{R}_{y}(1)
\end{array}\right] \overline{\mathbf{a}}=\mathbf{0} .
$$

We arrange the equations in (12) in terms of $\mathbf{a}$ as

$$
\mathbf{R}_{q} \mathbf{a}=\mathbf{r}_{q},
$$

where $\mathbf{R}_{q}$ is $\left(p_{2}+1\right) \times\left(p_{1} p_{2}+p_{1}+p_{2}\right)$ matrix and $\mathbf{r}_{q}$ is $\left(p_{2}+1\right)$ vector. 

obtain

Multiplying both sides of (10) by $\mathbf{R}_{q}$ and using (13), we

$$
\sigma_{v}^{2} \mathbf{R}_{q} \mathbf{R}_{y}^{-1} \mathbf{a}=\mathbf{r}_{q}-\mathbf{R}_{q} \mathbf{a}_{\mathrm{LS}} .
$$

The LS estimate of $\sigma_{v}^{2}$ can be obtained via

$$
\sigma_{v}^{2}=\frac{\left(\mathbf{R}_{q} \mathbf{R}_{y}^{-1} \mathbf{a}\right)^{T}\left(\mathbf{r}_{q}-\mathbf{R}_{q} \mathbf{a}_{\mathrm{LS}}\right)}{\left\|\mathbf{R}_{q} \mathbf{R}_{y}^{-1} \mathbf{a}\right\|^{2}},
$$

where $\|\cdot\|$ is the Euclidean norm. Now, we can estimate a and $\sigma_{v}^{2}$ by an iterative algorithm which is summarized in the following subsection.

3.2. The Proposed Algorithm. The proposed estimation algorithm can be summarized as follows.

Step 1. Estimate the autocorrelations $\widehat{r}_{y}(k, l) k=0, \ldots, p_{1}+1$, $l=0, \ldots, p_{2}$ using data samples as follows [15]:

$$
\begin{aligned}
\widehat{r}_{y}(k, l) & =\frac{1}{M N} \sum_{m=k+1}^{M} \sum_{n=l+1}^{N} y(m, n) y(m-k, n-l), \\
\widehat{r}_{y}(k, l) & =\widehat{r}_{y}(-k,-l), \\
\widehat{r}_{y}(k,-l) & =\widehat{r}_{y}(-k, l) \\
& =\frac{1}{M N} \sum_{m=1}^{M-k} \sum_{n=l+1}^{N} y(m, n) y(m+k, n-l) .
\end{aligned}
$$

Step 2. Form $\widehat{\mathbf{R}}_{y}, \widehat{\mathbf{r}}_{y}, \widehat{\mathbf{R}}_{q}$, and $\widehat{\mathbf{r}}_{q}$ and compute $\widehat{\mathbf{a}}_{\mathrm{LS}}=\widehat{\mathbf{R}}_{y}^{-1} \widehat{\mathbf{r}}_{y}$.

Step 3. Set $j=0$ and

$$
\widehat{\mathbf{a}}^{(j)}=\widehat{\mathbf{a}}_{\mathrm{LS}} .
$$

Step 4. Set $j=j+1$ and compute

$$
\begin{gathered}
\widehat{\sigma}_{v}^{2}=\frac{\left(\widehat{\mathbf{R}}_{q} \widehat{\mathbf{R}}_{y}^{-1} \widehat{\mathbf{a}}^{(j-1)}\right)^{T}\left(\widehat{\mathbf{r}}_{q}-\widehat{\mathbf{R}}_{q} \widehat{\mathbf{a}}_{\mathrm{LS}}\right)}{\left\|\widehat{\mathbf{R}}_{q} \widehat{\mathbf{R}}_{y}^{-1} \widehat{\mathbf{a}}^{(j-1)}\right\|^{2}} \\
\widehat{\mathbf{a}}^{(j)}=\widehat{\mathbf{a}}^{(j-1)}+\mu\left(\widehat{\mathbf{a}}_{\mathrm{LS}}+\widehat{\sigma}_{v}^{2} \widehat{\mathbf{R}}_{y}^{-1} \widehat{\mathbf{a}}^{(j-1)}-\widehat{\mathbf{a}}^{(j-1)}\right) .
\end{gathered}
$$

Step 5. If [13]

$$
\left\|\frac{\widehat{\mathbf{a}}^{(j)}-\widehat{\mathbf{a}}^{(j-1)}}{\widehat{\mathbf{a}}^{(j)}}\right\| \leq \delta,
$$

where $\delta$ is a small positive number, for example, $\delta=10^{-3}$, the convergence is achieved and the iteration process must be terminated; otherwise, go to Step 4.

3.3. Convergence Analysis. The convergence analysis of the proposed algorithm is similar to the method proposed in [21]. The following result discusses the convergence conditions of the proposed algorithm. In this analysis, the observation noise variance is assumed to be known.
Theorem 1. The necessary and sufficient condition for the convergence of the proposed algorithm is to require the step size parameter $\mu$ to satisfy the following condition:

$$
0<\mu<\frac{2}{1-\lambda_{\min }},
$$

where $\lambda_{\min }$ is the minimum eigenvalue of the $\sigma_{v}^{2} \mathbf{R}_{y}^{-1}$ matrix.

Proof. Defining estimation error matrix $\Delta \mathbf{a}^{(j)}=\mathbf{a}^{(j)}-\mathbf{a}$, substituting $\mathbf{a}-\mathbf{a}_{\mathrm{LS}}-\sigma_{v}^{2} \mathbf{R}_{y}^{-1} \mathbf{a}=\mathbf{0}$ into (11), and using $\Delta \mathbf{a}^{(j)}=$ $\mathbf{a}^{(j)}-\mathbf{a}$, we obtain

$$
\Delta \mathbf{a}^{(j)}=\mathbf{I}-\mu\left(\mathbf{I}-\sigma_{v}^{2} \mathbf{R}_{y}^{-1}\right) \Delta \mathbf{a}^{(j-1)} .
$$

The eigenvalues of $\mathbf{I}-\mu\left(\mathbf{I}-\sigma_{v}^{2} \mathbf{R}_{y}^{-1}\right)$ are $1-\mu\left(1-\lambda_{j}\right)$ where $\lambda_{j}=\lambda_{j}\left(\sigma_{v}^{2} \mathbf{R}_{y}^{-1}\right)$ is between 0 and 1 .

If $-1<1-\mu\left(1-\lambda_{j}\right)<1$, then $\lim _{i \rightarrow \infty} \Delta \mathbf{a}^{(j)}=0$. In this case, we have $0<\mu<2 /\left(1-\lambda_{j}\right)$, and their intersection is $0<\mu<2 /\left(\lambda_{\max }\left(\mathbf{I}-\sigma_{v}^{2} \mathbf{R}_{y}^{-1}\right)\right)=2 /\left(1-\lambda_{\text {min }}\right)$.

\section{Simulation Results}

In order to evaluate the performance of the proposed method and to compare with the Least-Squares (LS) method, two examples are presented. In the first example we generate data using a synthetic $2 \mathrm{D}$ noisy $\mathrm{AR}$ and in the second example we apply the methods for 2D sinusoidal spectral estimation.

Example 1. Consider a synthetic 2D noisy AR model as follows:

$$
\begin{aligned}
x(m, n)= & a(0,1) x(m, n-1)+a(0,2) x(m, n-2) \\
& +a(1,0) x(m-1, n)+a(1,1) x(m-1, n-1) \\
& +a(1,2) x(m-1, n-2) \\
& +a(2,0) x(m-2, n) \\
& +a(2,1) x(m-2, n-1) \\
& +a(2,2) x(m-2, n-2)+u(m, n), \\
& y(m, n)=x(m, n)+v(m, n),
\end{aligned}
$$

where the $u(m, n)$ and $v(m, n)$ are white Gaussian noises which are mutually uncorrelated.

The signal to noise ratio (SNR) is calculated by

$$
\mathrm{SNR}=10 \log _{10}\left(\frac{\sigma_{x}^{2}}{\sigma_{v}^{2}}\right) \mathrm{dB},
$$

where $\sigma_{x}^{2}$ is the variance of the signal $x(m ., n)$. We assume that $\sigma_{u}^{2}=1$ and $\sigma_{v}^{2}$ is adjusted to produce a value of the SNR. The $N$ is assumed to be equal to $M$. The step size, $\mu$, is set to one. 
TABLE 1: Simulation results $(J=100, N=128$, and SNR $=5 \mathrm{~dB})$.

\begin{tabular}{|c|c|c|c|c|}
\hline True value & $\begin{array}{c}\text { LS } \\
\text { mean of estimated } \\
\text { value }\end{array}$ & $\begin{array}{c}\text { LS } \\
\text { standard deviation of } \\
\text { estimated value }\end{array}$ & $\begin{array}{c}\text { Proposed } \\
\text { mean of estimated } \\
\text { value }\end{array}$ & $\begin{array}{c}\text { Proposed } \\
\text { standard deviation of } \\
\text { estimated value }\end{array}$ \\
\hline$a(0,1)=0.3$ & 0.2221 & 0.0088 & 0.2947 & 0.0217 \\
\hline$a(0,2)=0.2$ & 0.1602 & 0.0069 & 0.1957 & 0.0153 \\
\hline$a(1,0)=0.3$ & 0.2226 & 0.0073 & 0.2952 & 0.0246 \\
\hline$a(1,1)=-0.09$ & -0.0366 & 0.0084 & -0.0863 & 0.0223 \\
\hline$a(1,2)=-0.06$ & -0.0284 & 0.0082 & -0.0574 & 0.0137 \\
\hline$a(2,0)=0.2$ & 0.1602 & 0.0079 & 0.1955 & 0.0128 \\
\hline$a(2,1)=-0.06$ & -0.0284 & 0.0087 & -0.0565 & 0.0140 \\
\hline$a(2,2)=-0.04$ & -0.0217 & 0.0085 & -0.0387 & 0.0138 \\
\hline RMSE (\%) & \multicolumn{2}{|c|}{26.5437} & \multicolumn{2}{|c|}{6.1187} \\
\hline NIPT & \multicolumn{2}{|c|}{-} & \multicolumn{2}{|c|}{5.5400} \\
\hline
\end{tabular}

The methods are compared in terms of normalized root mean squared error (RMSE) which is defined by

$$
\operatorname{RMSE}=\frac{\sqrt{\sum_{j=1}^{J}\left\|\widehat{\mathbf{a}}_{j}-\mathbf{a}\right\|^{2} / J}}{\|\mathbf{a}\|},
$$

where $\widehat{\mathbf{a}}_{j}$ is the estimate of $\mathbf{a}$ in the $j$ th trial and $J$ is the total number of trials. The mean number of iterations per test (NIPT) for the proposed method is also presented against the criterion (19) with a choice of $\delta=0.001$.

In this example, we set $N=M=128, \mathrm{SNR}=5 \mathrm{~dB}$, and $J=$ 100. Results of simulations are summarized in Table 1. From Table 1, it can be seen that the performance of the proposed method is better than the LS method.

Example 2. Consider 2D sinusoidal signal in the presence of the noise as follows:

$$
\begin{gathered}
x(m, n)=a_{1} \cos \left(\omega_{1} m+\omega_{2} n+\phi_{1}\right), \\
y(m, n)=x(m, n)+w(m, n),
\end{gathered}
$$

where $\omega_{1}=1, \omega_{2}=2, \mathrm{rad} /$ sample $\phi_{1}$ is a random phase uniformly distributed over $[0,2 \pi]$, and $v(m, n)$ is zeromean Gaussian noise with variance $\sigma_{v}^{2}=1$. Based on linear prediction property of sinusoidal signals, we can model $x(m, n)$ as a $2 \mathrm{D}$ AR order $(2,2)$ with zero input driving noise $u(m, n)=0$. Then, we estimate the model parameters and compute the corresponding normalized power spectrum.

In this example, parameter $a_{1}$ is adjusted to produce SNR equal to $-10 \mathrm{~dB}$ and $N=M=128$. The mean of the spectrum is depicted in Figures 1 and 2 for the proposed and the LS methods, respectively.

It can be seen from figures of the power spectrum that the proposed method can estimate a spectrum sharper than that of the LS method. Note that, in all simulations presented in this section, the convergence of the proposed method was reached usually within a few iterations (NIPT $=6$ iterations on average).

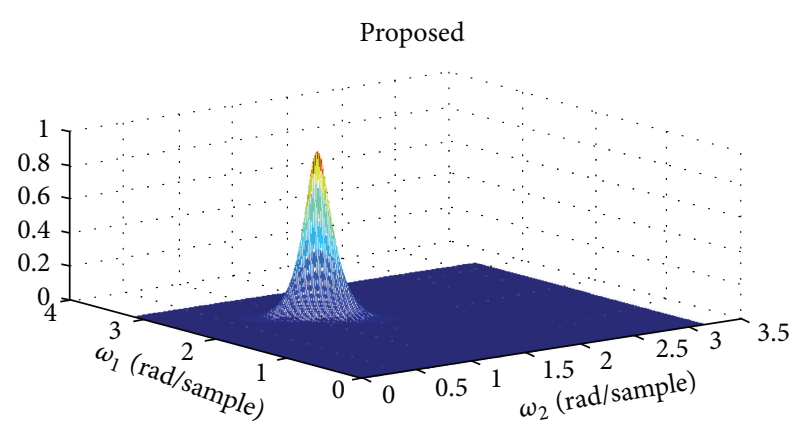

FIgURE 1: Mean of the normalized power spectrum, the proposed method, $N=128, \mathrm{SNR}=-10(\mathrm{~dB})$, and $J=100$.

LS

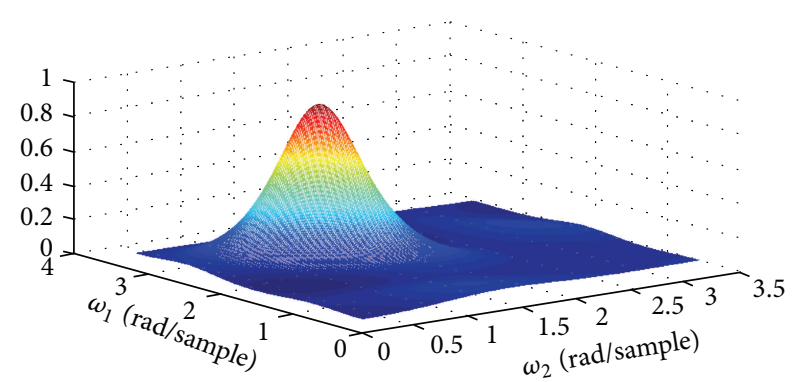

FIGURE 2: Mean of the normalized power spectrum, the LS method, $N=128, \mathrm{SNR}=-10(\mathrm{~dB})$, and $J=100$.

\section{Conclusion}

The two-dimensional noisy AR problem is addressed. The Yule-Walker equations are solved by using adaptive steepest descent algorithm. The induced bias from the observation noise variance is removed using the Yule-Walker equation beyond the order of the model. Simulation results showed that the proposed method can estimate frequency of sinusoidal signals sharper than that of the LS method. 


\section{Conflict of Interests}

The author declares that there is no conflict of interests regarding the publication of this paper.

\section{References}

[1] X. Zhang and X. Wu, "Image interpolation by adaptive 2-D autoregressive modeling and soft-decision estimation," IEEE Transactions on Image Processing, vol. 17, no. 6, pp. 887-896, 2008.

[2] S. Oe, "Texture segmentation method by using two-dimensional AR model and Kullback information," Pattern Recognition, vol. 26, no. 2, pp. 237-244, 1993.

[3] L. He, Z. Yu, Z. Gu, and Y. Li, "Long-tail distribution based multiscale-multiband autoregressive detection for hyperspectral imagery," Multidimensional Systems and Signal Processing, vol. 24, no. 1, pp. 65-85, 2013.

[4] R. R. Hansen Jr. and R. Chellappa, "Noncausal 2-D spectrum estimation for direction finding," IEEE Transactions on Information Theory, vol. 36, no. 1, pp. 108-125, 1990.

[5] M. Kay, V. Nagesha, and J. Salisbury, "Broad-band detection based on two-dimensional mixed autoregressive model," IEEE Transactions on Signal Processing, vol. 41, no. 7, pp. 2413-2428, 1993.

[6] S. M. Kay and S. B. Doyle, "Rapid estimation of the rangeDoppler scattering function," IEEE Transactions on Signal Processing, vol. 51, no. 1, pp. 255-268, 2003.

[7] D. Umansky and M. Pätzold, "A two-dimensional autoregressive model for MIMO wideband mobile radio channels," in Proceedings of the IEEE Global Telecommunications Conference (GLOBECOM '08), pp. 1-6, IEEE, New Orleans, La, USA, December 2008.

[8] A. H. Kayran and E. Camcioglu, "New efficient 2-D lattice structures for general autoregressive modeling of random fields," IEEE Transactions on Signal Processing, vol. 62, no. 6, pp. 15901602, 2014.

[9] B. Choi, "Model identification of a noncausal 2-D AR process using a causal 2-D AR model on the nonsymmetric half-plane," IEEE Transactions on Signal Processing, vol. 51, no. 5, pp. 14121421, 2003.

[10] B. Choi and D. N. Politis, "Modeling 2-D AR processes with various regions of support," IEEE Transactions on Signal Processing, vol. 55, no. 5, pp. 1696-1707, 2007.

[11] C. E. Davila, "A subspace approach to estimation of autoregressive parameters from noisy measurements," IEEE Transactions on Signal Processing, vol. 46, no. 2, pp. 531-534, 1998.

[12] A. Mahmoudi, M. Karimi, and H. Amindavar, "Parameter estimation of autoregressive signals in presence of colored AR(1) noise as a quadratic eigenvalue problem," Signal Processing, vol. 92, no. 4, pp. 1151-1156, 2012.

[13] W. X. Zheng, "Autoregressive parameter estimation from noisy data," IEEE Transactions on Circuits and Systems II: Analog and Digital Signal Processing, vol. 47, no. 1, pp. 71-75, 2000.

[14] A. Mahmoudi and M. Karimi, "Estimation of the parameters of multichannel autoregressive signals from noisy observations," Signal Processing, vol. 88, no. 11, pp. 2777-2783, 2008.

[15] S. Lee and T. Stathaki, "Two-dimensional autoregressive modelling using joint second and third order statistics and a weighting scheme," in Proceedings of the 12th European Signal Processing Conference (EUSIPCO '04), 2004.
[16] A. Mahmoudi, "Two dimensional autoregressive estimation from noisy observations as a quadratic eigenvalue problem," Multidimensional Systems and Signal Processing, 2014.

[17] B. Aksasse and L. Radouane, "Two-dimensional autoregressive (2-D AR) model order estimation," IEEE Transactions on Signal Processing, vol. 47, no. 7, pp. 2072-2077, 1999.

[18] X.-D. Zhang and J. Cheng, "High resolution two-dimensional ARMA spectral estimation," IEEE Transactions on Signal Processing, vol. 39, no. 3, pp. 765-770, 1991.

[19] S. M. Kay, Modern Spectral Estimation, Prentice-Hall, Englewood Cliffs, NJ, USA, 1988.

[20] S. Haykin, Adaptive Filter Theory, Prentice-Hall, Englewood Cliffs, NJ, USA, 2001.

[21] A. Mahmoudi, "Adaptive algorithm for multichannel autoregressive estimation in spatially correlated noise," Journal of Stochastics, vol. 2014, Article ID 502406, 7 pages, 2014. 


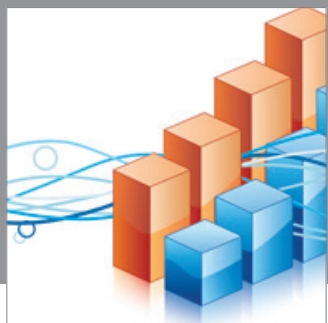

Advances in

Operations Research

mansans

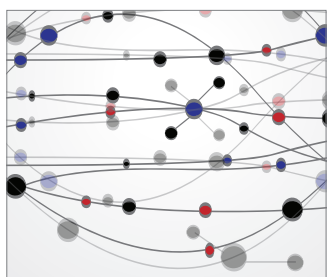

The Scientific World Journal
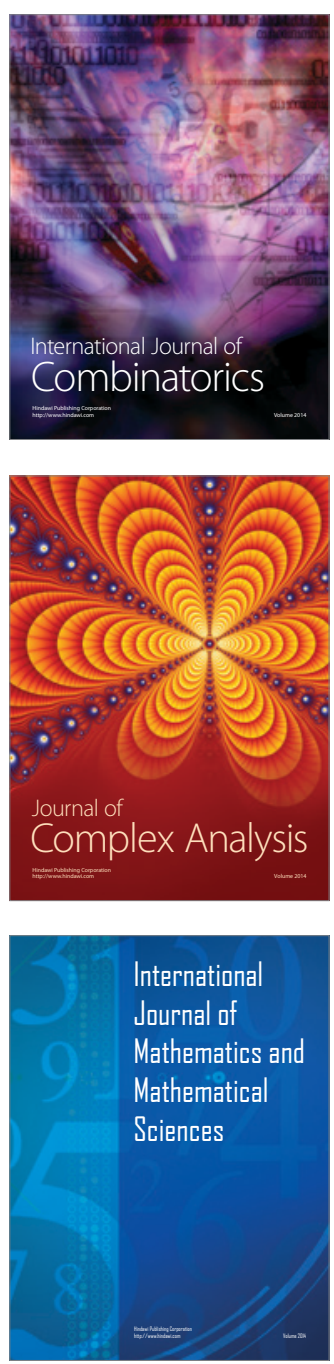
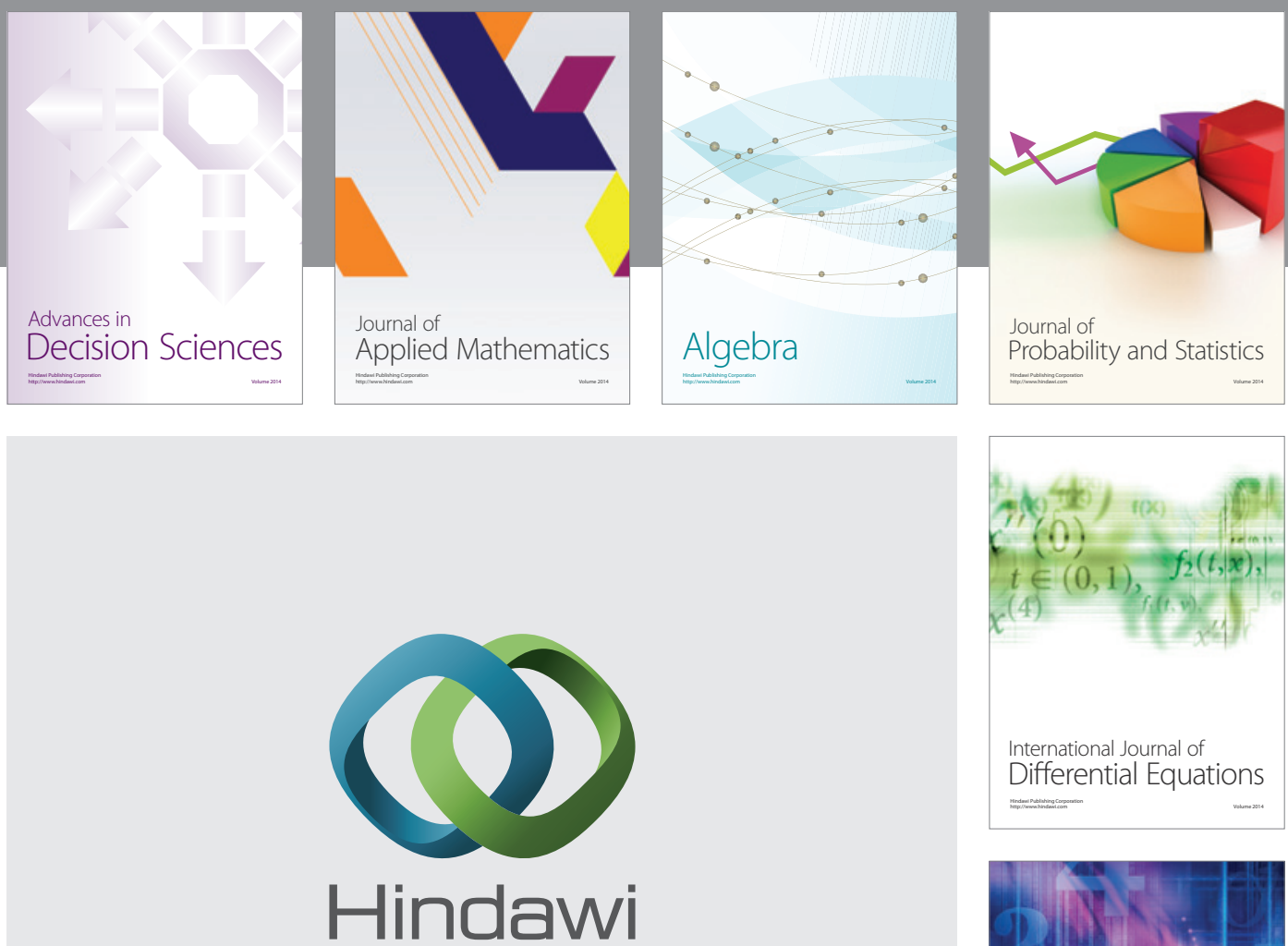

Submit your manuscripts at http://www.hindawi.com
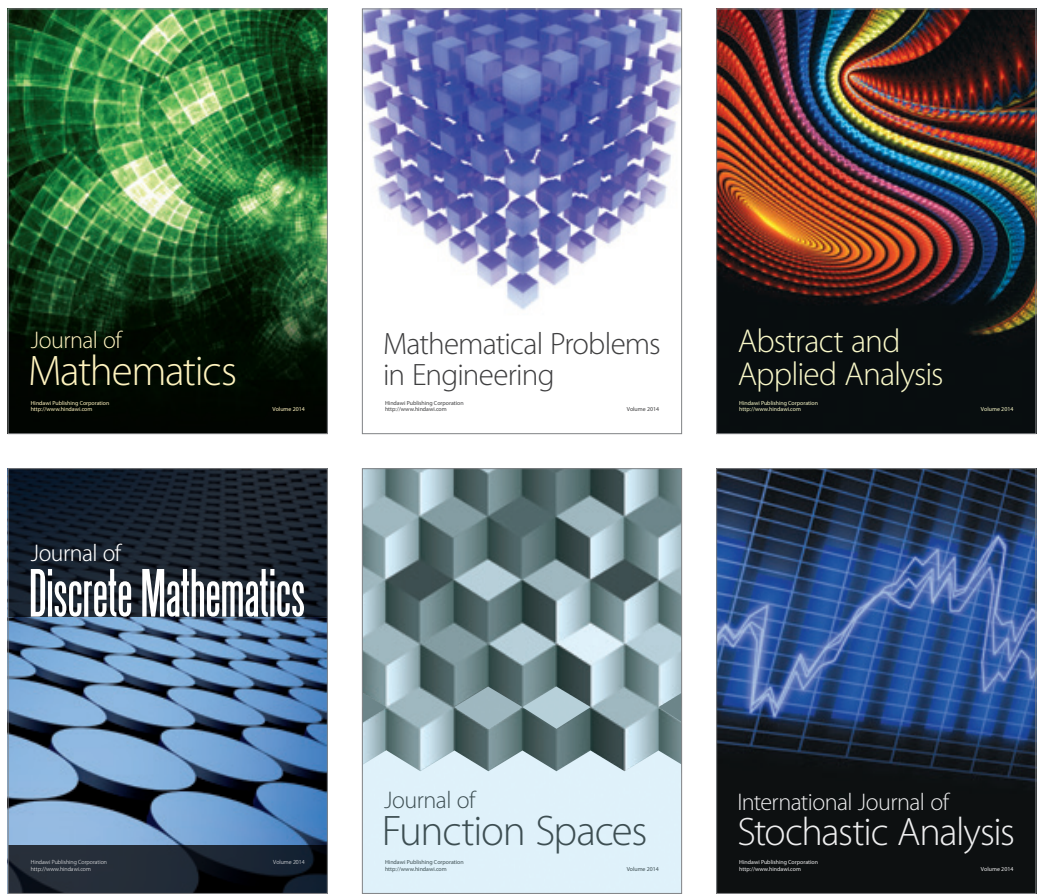

Journal of

Function Spaces

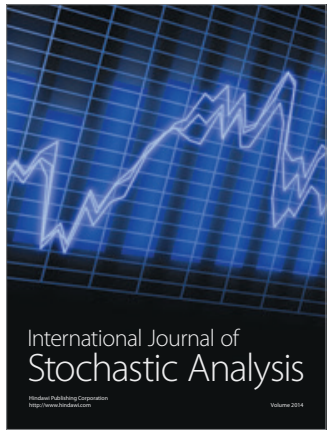

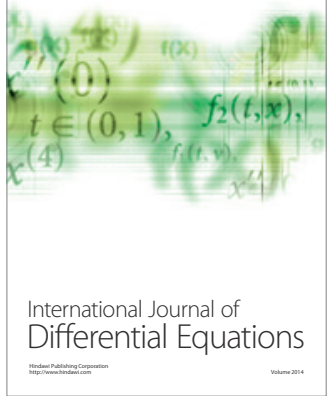
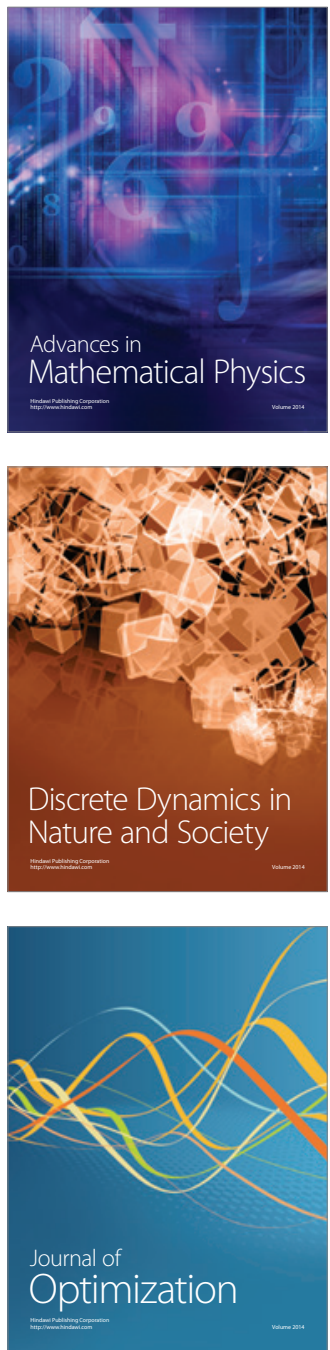